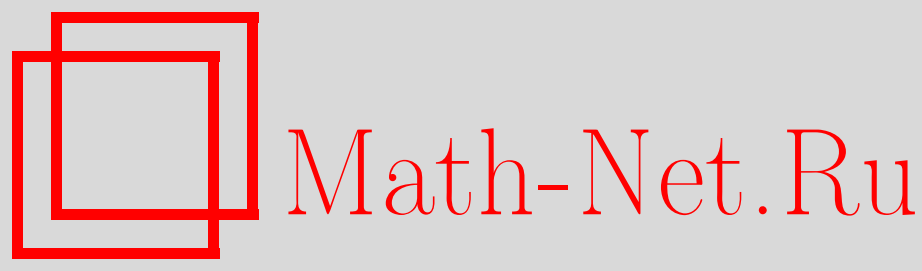

Л. Ц. Аджемян, С. Е. Воробьева, М. В. Компаниец, Представление несингулярными интегралами $\beta$ функции и аномальных размерностей в моделях критической динамики, ТМФ, 2015, том 185, номер 1, 3-11

DOI: https://doi.org/10.4213/tmf8911

Использование Общероссийского математического портала Math-Net.Ru подразумевает, что вы прочитали и согласны с пользовательским соглашением http://www . mathnet.ru/rus/agreement

Параметры загрузки:

IP : 18.209 .158 .208

26 апреля 2023 г., 14:08:40

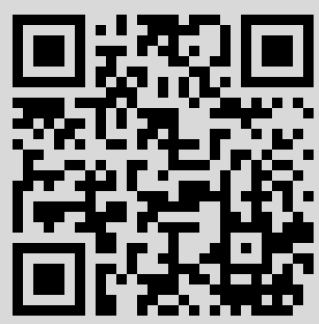


Том 185, № 1

октябрь, 2015

(C) 2015 г.

Л. Ц. Аджемян*, С. Е. Воробьева*, М. В. Компаниец*

\title{
ПРЕДСТАВЛЕНИЕ НЕСИНГУЛЯРНЫМИ ИНТЕГРАЛАМИ $\beta$ ФУНКЦИИ И АНОМАЛЬНЫХ РАЗМЕРНОСТЕЙ В МОДЕЛЯХ КРИТИЧЕСКОЙ ДИНАМИКИ
}

\begin{abstract}
Предложен способ вычисления $\beta$-функции и аномальных размерностей в моделях критической динамики, удобный для численных расчетов в рамках ренормгруппы и $\varepsilon$-разложения. Эти величины выражены через ренормированные функции Грина, ренормировка которых проведена с помощью $R$-операции, представленной в форме, позволяющей в явном виде произвести сокращение ультрафиолетовых расходимостей в диаграммах Фейнмана. Необходимые для расчета интегралы не содержат полюсов по $\varepsilon$ и удобны для проведения численного интегрирования.
\end{abstract}

Ключевые слова: ренормализационная группа, $\varepsilon$-разложение, многопетлевые диаграммы, критические показатели.

DOI: $10.4213 / \operatorname{tmf} 8911$

\section{1. ВВЕДЕНИЕ}

Метод ренормализационной группы (РГ) и $\varepsilon$-разложения является в настоящее время основным инструментом изучения как статических, так и динамических критических явлений [1]. Для статических критических показателей в настоящее время известны аналитические ответы вплоть до высоких порядков теории возмущений (вплоть до 5-го порядка $\varepsilon$-разложения в модели $\phi^{4}$, см., например, работы [2]). Аналитические расчеты в моделях критической динамики значительно менее развиты, они ограничиваются, как правило, первым порядком теории возмущений, лишь в отдельных случаях имеются результаты в более высоких порядках - во втором порядке в теории перколяции [3], в третьем порядке в $A$-модели [4]. Известны также численные результаты во втором порядке в $H$-модели [5] и теории турбулентности [6].

* Санкт-Петербургский государственный университет, Санкт-Петербург, Россия. E-mail: loran.adzhemyan@gmail.com 
В работах [7], [8] предложен подход, позволяющий сводить вычисление $\beta$-функции и аномальных размерностей в моделях критической статики к расчету интегралов, не содержащих сингулярностей по $\varepsilon$, и потому удобных для численного интегрирования. В работе [9] это позволило впервые провести полностью независимую проверку аналитических пятипетлевых результатов [2]. В настоящей работе такой подход обобщается на модели критической динамики. Рассмотрение проводится на примере $A$-модели.

\section{2. РЕНОРМИРОВКА МОДЕЛИ}

Рассмотрим $A$-модель критической динамики в пространстве размерности $d=$ $4-\varepsilon$. Неренормированное действие этой модели определяется набором $\phi_{0} \equiv\left\{\psi_{0}, \psi_{0}^{\prime}\right\}$ двух полей и имеет вид [1]

$$
\begin{aligned}
S_{0}\left(\phi_{0}\right) & =\lambda_{0} \psi_{0}^{\prime} \psi_{0}^{\prime}+\psi_{0}^{\prime}\left[-\partial_{t} \psi_{0}+\lambda_{0} \frac{\delta S_{0}^{\mathrm{st}}}{\delta \psi_{0}}\right]= \\
& =\lambda_{0} \psi_{0}^{\prime} \psi_{0}^{\prime}+\psi_{0}^{\prime}\left[\partial_{t} \psi_{0}+\lambda_{0}\left(\partial^{2} \psi_{0}-\tau_{0} \psi_{0}-\frac{1}{3 !} g_{0} \psi_{0}^{3}\right)\right]
\end{aligned}
$$

с неренормированным статическим действием

$$
S_{0}^{\mathrm{st}}\left(\phi_{0}\right)=-\frac{\left(\partial \psi_{0}\right)^{2}}{2}-\frac{\tau_{0} \psi_{0}^{2}}{2}-\frac{1}{4 !} g_{0} \psi_{0}^{4}
$$

Ренормированное действие дается выражением [1]

$$
S_{\mathrm{R}}=Z_{1} \lambda \psi^{\prime} \psi^{\prime}+\psi^{\prime}\left[-Z_{2} \partial_{t} \psi+\lambda\left(Z_{3} \Delta \psi-Z_{4} \tau \psi-\frac{1}{3 !} Z_{5} \mu^{\varepsilon} g \psi^{3}\right)\right],
$$

оно получается из исходного мультипликативной ренормировкой параметров и полей

$$
\lambda_{0}=\lambda Z_{\lambda}, \quad \tau_{0}=\tau Z_{\tau}, \quad g_{0}=g \mu^{\varepsilon} Z_{g}, \quad \psi_{0}=\psi Z_{\psi}, \quad \psi_{0}^{\prime}=\psi^{\prime} Z_{\psi^{\prime}},
$$

где

$$
\begin{gathered}
Z_{1}=Z_{\lambda} Z_{\psi^{\prime}}^{2}, \quad Z_{2}=Z_{\psi^{\prime}} Z_{\psi}, \quad Z_{3}=Z_{\psi^{\prime}} Z_{\lambda} Z_{\psi} \\
Z_{4}=Z_{\psi^{\prime}} Z_{\lambda} Z_{\tau} Z_{\psi}, \quad Z_{5}=Z_{\psi^{\prime}} Z_{\lambda} Z_{g} Z_{\psi}^{3} .
\end{gathered}
$$

С другой стороны, ренормированное действие может быть представлено в виде суммы базового действия $S_{\mathrm{B}}$ и контрчленов $\Delta S$ :

$$
S_{\mathrm{R}}=S_{\mathrm{B}}+\Delta S
$$

где базовое действие имеет вид

$$
S_{\mathrm{B}}=\lambda \psi^{\prime} \psi^{\prime}+\psi^{\prime}\left[-\partial_{t} \psi+\lambda\left(\triangle \psi-\tau \psi-\frac{1}{3 !} \mu^{\varepsilon} g \psi^{3}\right)\right] .
$$

Введем обозначение для 1-неприводимой функции Грина базовой теории (4):

$$
\Gamma_{\left(n_{1}, n_{2}\right)} \equiv\langle\underbrace{\psi \ldots \psi}_{n_{1}} \underbrace{\psi^{\prime} \ldots \psi^{\prime}}_{n_{2}}\rangle_{1 \text {-непр }}
$$


Поверхностные ультрафиолетовые (УФ) расходимости при $\varepsilon=0$ присутствуют в диаграммах 1-неприводимых функций $\Gamma_{(0,2)}, \Gamma_{(3,1)}$, имеющих логарифмическую расходимость, и в квадратично-расходящихся диаграммах функции $\Gamma_{(1,1)}$. Будем называть 1-неприводимые подграфы, которые имеют поверхностную УФ-расходимость, существенными. Очевидно, данные подграфы совпадают с диаграммами 1-неприводимых функций $\Gamma_{(0,2)}, \Gamma_{(3,1)}$ и $\Gamma_{(1,1)}$.

Учет контрчленов в (3) можно заменить действием $R$-операции на диаграммы базовой теории (4) [1] и записать ренормированные функции $\Gamma_{\left(n_{1}, n_{2}\right)}^{\mathrm{R}}$ в виде

$$
\Gamma_{\left(n_{1}, n_{2}\right)}^{\mathrm{R}}=R \Gamma_{\left(n_{1}, n_{2}\right)}=(1-K) R^{\prime} \Gamma_{\left(n_{1}, n_{2}\right)},
$$

где операция $R^{\prime}$ устраняет расходимости в существенных подграфах диаграммы, а $(1-K)$ - остающуюся поверхностную расходимость. Конкретная схема ренормировки определяется заданием операции $K$ отбора расходящихся частей 1 -неприводимых диаграмм.

Как и в статье [6], выберем схему ренормировки, аналогичную схеме вычитаний на нулевых импульсах (и частотах), с дополнительным условием $\mu^{2}=\tau$. Конкретизировать такой выбор удобно с помощью следующих нормированных функций (на нулевых частотах и импульсах):

$$
\begin{aligned}
& \bar{\Gamma}_{1}=\left.\frac{\Gamma_{(0,2)}}{2 \lambda}\right|_{p=0, \omega=0}, \\
& \bar{\Gamma}_{2}=\left.\partial_{i \omega} \Gamma_{(1,1)}\right|_{p=0, \omega=0}, \\
& \bar{\Gamma}_{3}=-\left.\frac{1}{2} \partial_{p}^{2} \frac{\Gamma_{(1,1)}}{\lambda}\right|_{p=0, \omega=0}, \\
& \bar{\Gamma}_{4}=-\left.\frac{\Gamma_{(1,1)}-\left.\Gamma_{(1,1)}\right|_{\tau=0}}{\lambda \tau}\right|_{p=0, \omega=0}, \\
& \bar{\Gamma}_{5}=-\left.\frac{\Gamma_{(3,1)}}{\lambda g \mu^{\varepsilon}}\right|_{p=0, \omega=0} .
\end{aligned}
$$

Константы ренормировки определим из требований

$$
\left.\bar{\Gamma}_{i}^{\mathrm{R}}\right|_{\mu^{2}=\tau}=1, \quad i=1, \ldots, 5 .
$$

Эти условия означают, что в точке нормировки контрчлены полностью сокращают диаграммные вклады в ренормированные аналоги функций $\bar{\Gamma}_{i}$.

Константам ренормировки $Z_{i}$, определяющимся условиями $(10)$, соответствует операция $K$ отбора расходящихся частей диаграмм:

$$
\begin{aligned}
K \Gamma_{(0,2)}= & \left.\Gamma_{(0,2)}\right|_{p=0, \omega=0, \mu^{2}=\tau}, \quad K \Gamma_{(3,1)}=\left.\Gamma_{(3,1)}\right|_{p=0, \omega=0, \mu^{2}=\tau}, \\
K \Gamma_{(1,1)}= & {\left[\left.\Gamma_{(1,1)}\right|_{\tau=0}+\frac{\tau}{\mu^{2}}\left(\left.\Gamma_{(1,1)}\right|_{\mu^{2}=\tau}-\left.\Gamma_{(1,1)}\right|_{\tau=0}\right)\right]_{p=0, \omega=0}+} \\
& +\left.p^{2} \partial_{p^{2}} \Gamma_{(1,1)}\right|_{p=0, \omega=0, \mu^{2}=\tau}+\left.i \omega \partial_{i \omega} \Gamma_{(1,1)}\right|_{p=0, \omega=0, \mu^{2}=\tau}
\end{aligned}
$$


При $\mu^{2}=\tau$ операция (12) упрощается,

$$
\begin{aligned}
\left.K \Gamma_{(1,1)}\right|_{\mu^{2}=\tau}= & \left.\Gamma_{(1,1)}\right|_{p=0, \omega=0, \mu^{2}=\tau}+ \\
& +\left.p^{2} \partial_{p^{2}} \Gamma_{(1,1)}\right|_{p=0, \omega=0, \mu^{2}=\tau}+\left.i \omega \partial_{i \omega} \Gamma_{(1,1)}\right|_{p=0, \omega=0, \mu^{2}=\tau},
\end{aligned}
$$

так что при $\mu^{2}=\tau$ все три операции в (11) и (13) сводятся к вычитанию на нулевых импульсах и частотах при $\mu^{2}=\tau$.

Полную $R$-операцию можно записать в виде [9]

$$
R \Gamma=\prod_{i}\left(1-K_{i}\right) \Gamma
$$

где произведение берется по всем существенным (имеющим поверхностную расходимость) подграфам диаграмм, входящих в Г, и диаграммам в целом (если у них есть поверхностная расходимость).

\section{3. УРАВНЕНИЯ РГ}

В используемой схеме ренормировки константы ренормировки зависят, как и в схеме минимальных вычитаний (MB), только от константы связи и от размерности пространства, поэтому уравнения ренормгруппы для 1-неприводимых функций $\Gamma_{\left(n_{1}, n_{2}\right.}^{\mathrm{R}}$ можно получить аналогично тому, как это делается в монографии [1] для схемы МВ. Введем обозначения

$$
e_{0}=\left\{g_{0}, m_{0}, \lambda_{0}, \mu\right\}, \quad e=\{g, m, \lambda, \mu\}
$$

для набора затравочных и ренормированных параметров. Для удобства мы включили в первый из них ренормировочную массу $\mu$, от которой затравочное действие не зависит. Формулы ренормировки для 1-неприводимых функций $\Gamma_{\left(n_{1}, n_{2}\right)}^{\mathrm{R}}$ имеют вид

$$
\Gamma_{\left(n_{1}, n_{2}\right)}^{(0)}\left(e_{0}\right)=Z_{\psi}^{-n_{1}} Z_{\psi^{\prime}}^{-n_{2}} \Gamma_{\left(n_{1}, n_{2}\right)}^{\mathrm{R}}(e) .
$$

Уравнения РГ для $\Gamma_{\left(n_{1}, n_{2}\right)}^{R}$ получаются из условия независимости $\Gamma_{\left(n_{1}, n_{2}\right)}^{(0)}\left(e_{0}\right)$ от $\mu$ :

$$
\widetilde{\mathcal{D}}_{\mu} \Gamma_{\left(n_{1}, n_{2}\right)}^{(0)}=0
$$

где $\left.\widetilde{\mathcal{D}}_{\mu} \equiv \mu \partial_{\mu}\right|_{m_{0}, g_{0}, \lambda_{0}}$. Для ренормированных функций $\Gamma_{\left(n_{1}, n_{2}\right)}^{\mathrm{R}}$ отсюда с учетом $(15)$ получаем

$$
\widetilde{\mathcal{D}}_{\mu}\left[\ln \Gamma_{\left(n_{1}, n_{2}\right)}^{\mathrm{R}}-n_{1} \ln Z_{\psi}-n_{2} \ln Z_{\psi^{\prime}}\right]=0,
$$

таким образом, положив

$$
\gamma_{\phi} \equiv \widetilde{\mathcal{D}}_{\mu} \ln Z_{\phi}
$$

находим

$$
\widetilde{\mathcal{D}}_{\mu} \Gamma_{\left(n_{1}, n_{2}\right)}^{\mathrm{R}}=\left(n_{1} \gamma_{\psi}+n_{2} \gamma_{\psi^{\prime}}\right) \Gamma_{\left(n_{1}, n_{2}\right)}^{\mathrm{R}} .
$$

Переходя в этом выражении к дифференцированию по ренормированным переменным е с помощью равенства

$$
\begin{aligned}
\left(\mathcal{D}_{\mu}\right)_{g_{0}, m_{0}, \lambda_{0}}=\left(\mathcal{D}_{\mu}\right)_{g, m, \lambda} & +\left(\mathcal{D}_{\mu} g\right)_{g_{0}, m_{0}, \lambda_{0}} \partial_{g}+ \\
& +\left(\mathcal{D}_{\mu} \tau\right)_{g_{0}, m_{0}, \lambda_{0}} \partial_{\tau}+\left(\mathcal{D}_{\mu} \lambda\right)_{g_{0}, m_{0}, \lambda_{0}} \partial_{\lambda}
\end{aligned}
$$


имеем

$$
\left(\mathcal{D}_{\mu}+\left(\widetilde{\mathcal{D}}_{\mu} g\right) \partial_{g}+\left(\widetilde{\mathcal{D}}_{\mu} \tau\right) \partial_{\tau}+\left(\widetilde{\mathcal{D}}_{\mu} \lambda\right) \partial_{\lambda}\right) \Gamma_{\left(n_{1}, n_{2}\right)}^{\mathrm{R}}=\left(n_{1} \gamma_{\psi}+n_{2} \gamma_{\psi^{\prime}}\right) \Gamma_{\left(n_{1}, n_{2}\right)}^{\mathrm{R}} .
$$

Вводя с учетом (1) РГ-функции

$$
\gamma_{\tau} \equiv \widetilde{\mathcal{D}}_{\mu} \ln Z_{\tau}, \quad \gamma_{\lambda} \equiv \widetilde{\mathcal{D}}_{\mu} \ln Z_{\lambda}, \quad \gamma_{g} \equiv \widetilde{\mathcal{D}}_{\mu} \ln Z_{g}, \quad \beta \equiv \widetilde{\mathcal{D}}_{\mu} g=-g\left(\varepsilon+\gamma_{g}\right),
$$

получаем из (17) искомое уравнение ренормгруппы:

$$
\left(\mathcal{D}_{\mu}+\beta \partial_{g}-\gamma_{\tau} \mathcal{D}_{\tau}-\gamma_{\lambda} \mathcal{D}_{\lambda}\right) \Gamma_{\left(n_{1}, n_{2}\right)}^{\mathrm{R}}=\left(n_{1} \gamma_{\psi}+n_{2} \gamma_{\psi^{\prime}}\right) \Gamma_{\left(n_{1}, n_{2}\right)}^{\mathrm{R}},
$$

где $\left.\mathcal{D}_{\mu} \equiv \mu \partial_{\mu}\right|_{\tau, \lambda, g},\left.\mathcal{D}_{\tau} \equiv \tau \partial_{\tau}\right|_{\lambda, \mu, g},\left.\mathcal{D}_{\lambda} \equiv \lambda \partial_{\lambda}\right|_{\tau, \mu, g}$.

Уравнения (19) позволяют обосновать критический скейлинг: условие $\beta\left(g_{*}\right)=0$ определяет значение заряда $g_{*}$ в неподвижной точке. Для устойчивой неподвижной точки подстановка $g=g_{*}$ в уравнения (19) превращает их в уравнения Эйлера для однородных функций, а величины $\gamma_{\tau}^{*} \equiv \gamma_{\tau}\left(g_{*}\right)$ и $\gamma_{\phi}^{*} \equiv \gamma_{\phi}\left(g_{*}\right)$ определяют критические показатели соответствующих величин. Необходимые для расчета этих показателей РГ-функции $\beta$ и $\gamma_{i}$ находят обычно по теории возмущений, вычисляя константы ренормировки. Как следует из (18), РГ-функции выражаются через них с помощью соотношений

$$
\beta(g)=-\varepsilon \frac{g}{1+g \partial_{g} \ln Z_{g}}, \quad \gamma_{i}(g)=-\varepsilon \frac{g \partial_{g} \ln Z_{i}}{1+g \partial_{g} \ln Z_{g}} .
$$

Теория ренормировок гарантирует, что РГ-функции (20) УФ-конечны: полюсы по $\varepsilon$ в правых частях этих соотношений сокращаются. Однако сингулярный характер констант ренормировки весьма затрудняет их определение численными методами. Отсутствие полюсов в РГ-функциях (20) можно показать, используя уравнения (19): ренормированные функции $\Gamma_{\left(n_{1}, n_{2}\right)}^{\mathrm{R}}$ УФ-конечны по построению [1]. Выбрав достаточное число уравнений из (19), можно выразить через них РГ-функции, что и доказывает конечность последних. Мы используем этот прием для численного расчета РГ-функций через $\Gamma_{\left(n_{1}, n_{2}\right)}^{\mathrm{R}}$, точнее, через ренормированные аналоги функций $\bar{\Gamma}_{i}$ из (5)-(9):

$$
\bar{\Gamma}_{i}^{\mathrm{R}}=R \bar{\Gamma}_{i}, \quad i=1, \ldots, 5 .
$$

Уравнения для $\bar{\Gamma}_{1}^{\mathrm{R}}, \bar{\Gamma}_{4}^{\mathrm{R}}, \bar{\Gamma}_{5}^{\mathrm{R}}$ непосредственно получаются из (19) подстановкой их определений $(5),(8),(9)$, а для $\bar{\Gamma}_{3}^{\mathrm{R}}, \bar{\Gamma}_{4}^{\mathrm{R}}$ - действием на соответствующие уравнения из (19) операциями $\left.\partial_{p}^{2}(\cdot)\right|_{p=0, \omega=0}$ и $\left.\partial_{i} \omega(\cdot)\right|_{p=0, \omega=0}$ с использованием коммутативности этих операций и $R$-операции. Учитывая вытекающие из (2) и (16) связи между РГ-функциями

$$
\begin{gathered}
\gamma_{1}=\gamma_{\lambda}+2 \gamma_{\psi^{\prime}}, \quad \gamma_{2}=\gamma_{\psi}+\gamma_{\psi^{\prime}}, \quad \gamma_{3}=\gamma_{\lambda}+\gamma_{\psi^{\prime}}+\gamma_{\psi}, \\
\gamma_{4}=\gamma_{\lambda}+\gamma_{\tau}+\gamma_{\psi^{\prime}}+\gamma_{\psi}, \quad \gamma_{5}=\gamma_{\lambda}+\gamma_{g}+\gamma_{\psi^{\prime}}+3 \gamma_{\psi},
\end{gathered}
$$

получаем

$$
\left(\mu \partial_{\mu}+\beta \partial_{g}-\gamma_{\tau} \tau \partial_{\tau}-\gamma_{i}\right) \bar{\Gamma}_{i}^{\mathrm{R}}=0, \quad i=1, \ldots, 5 .
$$

Безразмерные величины $\bar{\Gamma}_{i}^{\mathrm{R}}$ зависят только от безразмерного отношения $\tilde{\tau}=\tau / \mu^{2}$, т. е. $\bar{\Gamma}_{i}^{\mathrm{R}}(\tau, \mu, \lambda)=\bar{\Gamma}_{i}^{\mathrm{R}}(\tilde{\tau})$. С учетом этого факта уравнения $(22)$ можно записать в виде

$$
\left(\beta \partial_{g}-\left(2+\gamma_{\tau}\right) \tilde{\tau} \partial_{\tilde{\tau}}-\gamma_{i}\right) \bar{\Gamma}_{i}^{\mathrm{R}}=0, \quad i=1, \ldots, 5, \quad \tilde{\tau} \equiv \frac{\tau}{\mu^{2}} .
$$


Переходя в этих уравнениях к точке нормировки $\tilde{\tau}=1$ и учитывая условие (10), получаем

$$
\left(2+\gamma_{\tau}\right) F_{i}=\gamma_{i}, \quad i=1, \ldots, 5
$$

где

$$
F_{i} \equiv \hat{\partial}_{\tilde{\tau}} \bar{\Gamma}_{i}^{\mathrm{R}}, \quad \hat{\partial}_{\tilde{\tau}}(\cdot) \equiv-\left.\partial_{\tilde{\tau}}(\cdot)\right|_{\tilde{\tau}=1} \cdot
$$

Подставляя в уравнения (23) вытекающее из $(21)$ соотношение $\gamma_{\tau}=\gamma_{4}-\gamma_{3}$, получим уравнения

$$
\left(2+\gamma_{4}-\gamma_{3}\right) F_{i}=\gamma_{i}, \quad i=1, \ldots, 5 .
$$

Решая эти уравнения для случаев $i=3,4$ относительно $\gamma_{3}$ и $\gamma_{4}$, а затем решая оставшиеся уравнения относительно $\gamma_{1}, \gamma_{2}$ и $\gamma_{5}$, находим

$$
\gamma_{i}=\frac{2 F_{i}}{1+F_{3}-F_{4}}, \quad i=1, \ldots, 5 .
$$

Эти равенства дают выражения для РГ-функции через ренормированные 1-неприводимые функции $F_{i}$. Однако, как и в статическом случае [6], [7], данные функции неудобны для вычислений, более удобными для расчета являются функции

$$
f_{i}=R \hat{\partial}_{\tilde{\tau}} \bar{\Gamma}_{i}
$$

в этих функциях $R$-операция действует на продифференцированные диаграммы, в которых уже положено $\mu^{2}=\tau(\tau=1)$, поэтому операция $K$ имеет простой вид (13). В то же время для функций $F_{i}$ из $(23)$ необходимо сначала продифференцировать ренормированную диаграмму, в которой операция $K$ дается выражением (12), и лишь затем перейти в точку нормировки $\mu^{2}=\tau$. Мы покажем ниже, что величины $F_{i}$ и $f_{i}$ связаны простыми соотношениями

$$
F_{i}=\left(1-F_{4}\right) f_{i}, \quad i=1,2,3,5
$$

Записывая уравнения (25) в виде

$$
\gamma_{i}=\frac{2 F_{i} /\left(1-F_{4}\right)}{1+F_{3} /\left(1-F_{4}\right)}
$$

и подставляя в них $f_{i}=F_{i} /\left(1-F_{4}\right)$ из $(27)$, приходим к соотношениям

$$
\gamma_{i}=\frac{2 f_{i}}{1+f_{3}}, \quad i=1,2,3,5,
$$

с помощью которых и будут производиться дальнейшие расчеты РГ-функций.

Используя соотношения (28) и (21), получаем формулы для вычисления остальных РГ-функций:

$$
\begin{aligned}
\gamma_{\lambda} & =\frac{2\left(f_{3}-f_{2}\right)}{1+f_{3}}, & \gamma_{\psi} & =\frac{f_{2}-f_{1}+f_{3}}{1+f_{3}} \\
\gamma_{\psi^{\prime}} & =\frac{f_{1}-f_{3}+f_{2}}{1+f_{3}}, & \gamma_{g} & =\frac{2\left(f_{5}-2 f_{3}-f_{2}+f_{1}\right)}{1+f_{3}} .
\end{aligned}
$$




\section{4. ПРЕДСТАВЛЕНИЕ R-ОПЕРАЦИИ}

Величины $f_{i}$ вычисляются в точке нормировки $\mu^{2}=\tau$, операция вычитания $1-K$ в формуле (14) для них сводится к вычитанию начального отрезка ряда по импульсу и частоте. Для логарифмических подграфов имеем

$$
(1-K) \Gamma\left(p^{2}, \omega\right)=\Gamma\left(p^{2}, \omega\right)-\left.\Gamma\right|_{p=0, \omega=0},
$$

а для квадратичных имеем

$$
(1-K) \Gamma\left(p^{2}, \omega\right)=\Gamma\left(p^{2}, \omega\right)-\left.\Gamma\right|_{p=0, \omega=0}-\left.p^{2} \partial_{p^{2}} \Gamma\right|_{p=0, \omega=0}-\left.i \omega \partial_{i \omega} \Gamma\right|_{p=0, \omega=0} .
$$

В обоих случаях операцию вычитания можно записать в виде остаточного члена ряда Тейлора в интегральной форме: для логарифмических подграфов

$$
(1-K) \Gamma\left(p^{2}, \omega\right)=\int_{0}^{1} d a \partial_{a} \Gamma\left(a p^{2}, a \omega\right),
$$

для квадратичных

$$
(1-K) \Gamma\left(p^{2}, \omega\right)=\int_{0}^{1} d a(1-a) \partial_{a}^{2} \Gamma\left(a p^{2}, a \omega\right) .
$$

Действие операции $R$ на диаграмму х с учетом соотношений (29), (30) можно записать в виде [9]

$$
R \chi=\prod_{i} \int_{0}^{1} d a_{i}\left(1-a_{i}\right)^{n_{i}} \partial_{a_{i}}^{n_{i}+1} \chi(\{a\}),
$$

где произведение берется по всем существенным подграфам $\chi^{(i)}$ (включая диаграмму $\chi$ как целое), $a_{i}$ - параметр растяжения внутри $i$-го подграфа импульсов и частот, втекающих в этот подграф, $n_{i}=0$ для логарифмических подграфов и $n_{i}=1$ для квадратичных. Преимущество такой записи ренормированных величин состоит в том, что ответ представляется в виде интегралов, конечных при $\varepsilon=0$, причем в форме, в которой не происходит сокращения больших вкладов в подынтегральном выражении (“теория без расходимостей”).

\section{5. РЕНОРМИРОВКА ВЕЛИЧИН $f_{i}$ И ИХ СВЯЗЬ С $F_{i}$}

Докажем соотношение (27). Рассмотрим с этой целью процедуру ренормировки в соотношении $(26)$. Операция $-\partial_{\tau}$, действуя на диаграммы базовой теории, сводится к сумме вставок во всевозможные линии составного оператора $\psi \psi^{\prime} \equiv \Psi$. Вставка такого оператора улучшает УФ-сходимость диаграмм, она порождает также новый тип расходящихся подграфов. Обозначим через $\Gamma_{\left(n_{1}, n_{2} ; 1\right)} 1$-неприводимую функцию, получающуюся вставкой одного оператора $\Psi$ в $\Gamma_{\left(n_{1}, n_{2}\right)}$, тогда

$$
-\partial_{\tau} \Gamma_{\left(n_{1}, n_{2}\right)}=\Gamma_{\left(n_{1}, n_{2} ; 1\right)} \text {. }
$$

Среди величин $\Gamma_{\left(n_{1}, n_{2} ; 1\right)}$ поверхностная расходимость (логарифмическая) остается только у $\Gamma_{(1,1 ; 1)}$. Соответствующие подграфы принадлежат, таким образом, классу существенных, и их надо учитывать в $R$-операции (14), принимая во внимание, что

$$
K \Gamma_{(1,1 ; 1)}=\left.\Gamma_{(1,1 ; 1)}\right|_{p=0, \omega=0, \mu^{2}=\tau} .
$$


Как и для величин $\bar{\Gamma}_{i}$, вычитания с $K$, взятым из $(33)$, приводят к тому, что в точке нормировки $p=0, \omega=0, \mu^{2}=\tau$ все диаграммные вклады в $\Gamma_{(1,1 ; 1)}^{\mathrm{R}}$ в ренормированной функции сокращаются контрчленами, и остается только беспетлевой вклад. С учетом того что такой вклад в $\Gamma_{(1,1)}$ равен $-p^{2}-\tau$, соответствующий вклад в $\Gamma_{(1,1 ; 1)}=-\partial_{\tau} \Gamma_{(1,1)}$ равен единице. Поэтому

$$
\left.\Gamma_{(1,1 ; 1)}^{\mathrm{R}}\right|_{p=0, \omega=0, \mu^{2}=\tau}=1 .
$$

Для неренормированных функций соотношение, аналогичное (32), имеет вид

$$
-\partial_{\tau_{0}} \Gamma_{\left(n_{1}, n_{2}\right)}^{(0)}\left(e_{0}, p\right)=\Gamma_{\left(n_{1}, n_{2} ; 1\right)}^{(0)}\left(e_{0}, p\right),
$$

где $\Gamma_{\left(n_{1}, n_{2} ; 1\right)}^{(0)}-1$-неприводимая функция со вставкой одного оператора $\psi_{0} \psi_{0}^{\prime} \equiv \Psi_{0}$. Перейдем в (35) к ренормированным величинам. Ренормировка составного оператора $\Psi$ определяется равенством $\Psi=Z_{\Psi} \Psi^{\mathrm{R}}$, с учетом этого ренормировка функции $\Gamma_{\left(n_{1}, n_{2} ; 1\right)}^{(0)}$ записывается в виде

$$
\Gamma_{\left(n_{1}, n_{2} ; 1\right)}^{(0)}=Z_{\psi}^{1-n_{1}} Z_{\psi^{\prime}}^{1-n_{2}} Z_{\Psi} \Gamma_{\left(n_{1}, n_{2} ; 1\right)}^{\mathrm{R}} .
$$

Выразим операцию $\partial_{\tau_{0}}$ в левой части (35) в терминах ренормированных переменных. В силу соотношений (1) фиксация переменных $g_{0}, \mu, \lambda_{0}$ означает также фиксацию $g, \mu, \lambda$. Таким образом,

$$
\left(\partial_{\tau_{0}}\right)_{g_{0}, \mu, \lambda_{0}}=Z_{\tau}^{-1}\left(\partial_{\tau}\right)_{g, \mu, \lambda} .
$$

Подставляя это равенство в (35) и используя формулы ренормировки (15), (36), получаем

$$
-\partial_{\tau} \Gamma_{\left(n_{1}, n_{2}\right)}^{\mathrm{R}}(e, p)=Z_{\psi} Z_{\psi^{\prime}} Z_{\tau} Z_{\Psi} \Gamma_{\left(n_{1}, n_{2} ; 1\right)}^{\mathrm{R}}(e, p)
$$

или, используя (2),

$$
-\partial_{\tau} \Gamma_{\left(n_{1}, n_{2}\right)}^{\mathrm{R}}(e, p)=\frac{Z_{4}}{Z_{\lambda}} Z_{\Psi} \Gamma_{\left(n_{1}, n_{2} ; 1\right)}^{\mathrm{R}}(e, p) .
$$

Учет констант ренормировки при построении функции $\Gamma_{\left(n_{1}, n_{2} ; 1\right)}^{\mathrm{R}}$ также можно заменить действием $R$-операции на диаграммы базовой теории [1]:

$$
\Gamma_{\left(n_{1}, n_{2} ; 1\right)}^{\mathrm{R}}=R \Gamma_{\left(n_{1}, n_{2} ; 1\right)} .
$$

Подставляя соотношения (38), (32) в (37), находим

$$
-\partial_{\tau} \Gamma_{\left(n_{1}, n_{2}\right)}^{\mathrm{R}}=-\frac{Z_{4}}{Z_{\lambda}} Z_{\Psi} R \partial_{\tau} \Gamma_{\left(n_{1}, n_{2}\right)} .
$$

Переписывая эти равенства для нормированных функций $\bar{\Gamma}_{i}$, заданных в $(5)-(9)$, учитывая формулы $(24),(26),(34)$ и полагая $\mu^{2}=\tau$, получаем

$$
1-F_{4}=\frac{Z_{4}}{Z_{\lambda}} Z_{\Psi}
$$

И

$$
F_{i}=\frac{Z_{4}}{Z_{\lambda}} Z_{\Psi} f_{i}, \quad i=1,2,3,5 .
$$

Подставляя в эти выражения величину $\left(Z_{4} / Z_{\lambda}\right) Z_{\Psi}$ из $(39)$, приходим к искомому равенству (27). 


\section{6. ЗАКЛЮЧЕНИЕ}

В представленной работе проведено обобщение результатов, полученных в работах [5] и [6] для моделей критической статики, на динамические модели. В работе [5] соотношение вида (28) в модели $\phi^{3}$ было угадано и проверено в четырехпетлевом приближении, в статье [6] это соотношение было доказано в произвольном порядке теории возмущений. Приведенное в настоящей работе доказательство соотношения (28) заметно короче, чем в [6]. Это связано с тем, что в нем используется информация о возможности обобщения процедуры ренормировки на случай расширенной теории с учетом составных операторов [10], а также ее формулировки в терминах $R$-операции [1].

Соотношения (26), (28), (31) позволяют вычислять РГ-функции $\beta$ и $\gamma_{i}$ непосредственно по диаграммам для $\Gamma_{(2,0)}, \Gamma_{(1,1)}$ и $\Gamma_{(3,1)}$ в форме, не содержащей полюсов по $\varepsilon$. Эффективность такого подхода была проверена путем численного расчета критических показателей в трехпетлевом приближении. Результат с большой точностью совпал с аналитическим ответом, приведенным в [1], [4]. Были рассчитаны также критические показатели в другой модели критического поведения - теории перколяции, в которой в настоящее время известен аналитический ответ во втором порядке $\varepsilon$-разложения [2]. В этом случае также имело место хорошее совпадение численных результатов с аналитическими. Предложенный способ может использоваться в старших порядках теории возмущений.

Благодарности. Работа выполнена при поддержке Санкт-Петербургского государственного университета (грант № 11.38.185.2014).

\section{Список литературы}

[1] А.Н. Васильев, Квантовополевая ренормгруппа в теории критического поведения и стохастической динамике, ПИЯФ, СПб., 1998.

[2] K. G. Chetyrkin, A. L. Kataev, F. V. Tkachev, Phys. Lett. B, 99:2 (1981), 147-150; Erratum 101:1 (1981), 457-458; K. G. Chetyrkin, S. G. Gorishny, S. A. Larin, F. V. Tkachov, Phys. Lett. B, 132:4-6 (1983), 351-354; Preprint P-0453, INR, Moscow, 1986; D. I. Kazakov, Phys. Lett. B, 133:6 (1983), 406-410; Д.И. Казаков, ТМФ, 58:3 (1984), 343-353; H. Kleinert, J. Neu, V. Shulte-Frohlinde, K. G. Chetyrkin, S. A. Larin, Phys. Lett. B, 272:1-2 (1991), 39-44; Erratum 319:4 (1993), 545.

[3] H.-K. Janssen, U. C. Täuber, Ann. Phys., 315:1 (2005), 147-192, arXiv: cond-mat/0409670.

[4] Н. В. Антонов, А. Н. Васильев, ТМФ, 60:1 (1984), 59-71.

[5] Л. Ц. Аджемян, А. Н. Васильев, Ю. С. Кабриц, М. В. Компаниец, ТМФ, 119:1 (1999), $73-92$.

[6] L. Ts. Adzhemyan, N. V. Antonov, M. V. Kompaniets, A. N. Vasil'ev, Internat. J. Modern Phys. B, 17:10 (2003), 2137-2170.

[7] Л. Ц. Аджемян, М. В. Компаниец, ТМФ, 169:1 (2011), 100-111.

[8] Л. Ц. Аджемян, М. В. Компаниец, С. В. Новиков, В. К. Сазонов, ТМФ, 175:3 (2013), $325-336$.

[9] L. Ts. Adzhemyan, M. V. Kompaniets, J. Phys.: Conf. Ser., 523 (2014), 012049, arXiv: 1309.5621.

[10] О. И. Завьялов, Перенормированные диаграммы Фейнмана, Наука, М., 1979.

[11] J. Zinn-Justin, Quantum Field Theory and Critical Phenomena, International Series of Monographs on Physics, 77, Oxford Univ. Press, Oxford, 1989. 